\title{
Review of: "A full pipeline of diagnosis and prognosis the risk of chronic diseases using deep learning and Shapley values: The Ravansar county anthropometric cohort study"
}

\author{
María Teresa García-Ordás ${ }^{1}$ \\ 1 Universidad de León \\ Potential competing interests: The author(s) declared that no potential competing interests exist.
}

In this paper, the authors describe a method for the detection of type 2 diabetes in a group of patients based on anthropometric characteristics and using deep learning techniques. The main aim is to find the factors affecting this disease and the intensity of the impact of each for precautionary measures. A priori, the work is interesting, but there are some aspects that need to be improved:

- It must be made clear in the abstract and the introduction that this work is only focused on type 2 diabetes, and not on other diseases such as hypertension, cardiovascular disease, heart attack and stroke. - In the abstract there are some typos that should be reviewed and perhaps it would be good to add a percentage to get an idea of how well the proposed method works.

- At the end of the introduction it is convenient to add a paragraph explaining what the structure of the article will be and what will be detailed in each section.

- It is necessary to give examples of what the data is like, details of how it has been extracted. In short, the dataset must be defined in detail, maybe in a new section.

- The data set, as the authors point out, is highly unbalanced. To solve this problem, weighting and oversampling techniques are used. It would be nice to use some known method like SMOTE or ADASYN, since its effectiveness is proven in numerous scientific articles.

- There are currently many published works for the detection of chronic diseases such as type 2 diabetes using novel artificial intelligence techniques. The authors must update the state of the art taking into account the many advances in the field.

(1- Diabetes detection using deep learning techniques with oversampling and feature augmentation - María Teresa García-Ordás, Carmen Benavides, José Alberto Benítez-Andrades, Héctor Alaiz-Moretón, Isaías García-Rodríguez

2- Stacking-based multi-objective evolutionary ensemble framework for prediction of diabetes mellitus

- N. Singh, P. Singh) 